\title{
Determinación de los tejidos y medios de elección para la confirmación microbiológica de los resultados serológicos de las campañas de control de Brucella melitensis en el ganado ovino
}

\author{
Determination of best tissues and culture media for microbiological confirmation \\ of the serological results of the control campaigns of Brucella melitensis in sheep

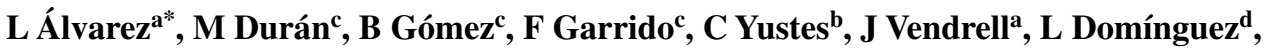 JF Fernández-Garayzabal ${ }^{d}$, AI Vela ${ }^{d}$, MV Latre ${ }^{\mathrm{e}}$ \\ aDepartamento de Ingeniería Agroalimentaria y Biotecnología, Universidad Politécnica de Cataluña. \\ ${ }^{\text {b}}$ Laboratorio de Salud Ambiental y Alimentaria. Departamento de Salud, Generalitat de Catalunya. \\ 'Laboratorio Central de Veterinaria de Santa Fe, Centro Nacional de Referencia de Brucelosis Animales, \\ Ministerio de Agricultura, Pesca y Alimentacion, Santa Fe, España. \\ ${ }^{\mathrm{d}}$ Departamento de Sanidad Animal, Universidad Complutense, Madrid, España. \\ eDepartamento de Patología Animal, Universidad de Zaragoza, Zaragoza, España.
}

\section{SUMMARY}

\begin{abstract}
Slaughtering seropositive animals in order to control ovine brucellosis requires a microbiological culture as a confirmatory technique. It is necessary to establish the most adequate tissues, due to difficulties involved in performing microbiological techniques in a large number of animals and samples. In this sense, there is a lack of data obtained from naturally infected sheep representatives of the different epidemiological situations, as recommended by OIE. In our study we carried out the necropsy of 92 ewes suspicious to be infected by Brucella and we performed microbiological culture and identification of 10 tissues per animal. Animals were classified according to an epidemiological survey as belonging to chronic brucellosis flocks, active brucellosis flocks and very low prevalence flocks. Results showed differences between flock types and also indicated that the analysis of samples from mammary gland and its lymph nodes was the best way to confirm infection in a suspicious flock. The combined use of Farrell and Thayer Martin media showed the best efficacy.
\end{abstract}

Palabras clave: brucelosis, Brucella melitensis, medio Farrell, medio Thayer Martin.

Key words: animal brucellosis, Brucella melitensis, Farrell medium, Thayer Martin medium.

\section{INTRODUCCIÓN}

La brucelosis es una de las zoonosis más importantes y se distribuye por todo el planeta con especial relevancia en el Área Mediterránea, Oriente Medio, América Latina y Asia (Corbel 1997, European Union 2001, Orduña y col 2001). Los Planes de Lucha y Erradicación están basados en la identificación de animales reaccionantes positivos a distintas técnicas serológicas y su posterior sacrificio. Con el objetivo de confirmar los resultados serológicos obtenidos en las campañas de control, se hace indispensable recurrir a las técnicas microbiológicas, ya que está generalmente aceptado que tan sólo el aislamiento del microorganismo es una prueba unívoca de la presencia de infección real (European Union 2001). Un problema adicional es la presencia de rebaños aparentemente no infectados, sin abortos, en los que se detectan animales positivos a fijación del complemento (FC). Por tanto es

Aceptado: 25.11.2009.

* Campus del Baix Llobregat, Edificio D4, Av. del Canal Olímpic, s/n.08860 Castelldefels, España; lorenzo.alvarez@upc.edu necesaria la confirmación real de la infección para evitar la pérdida de la calificación de "indemne" tanto a nivel de rebaño como regional.

Aunque los aislamientos obtenidos en infecciones experimentales han sido estudiados por distintos autores (Fensterbank y col 1982, Verger y col 1995, Durán 1998, Durán y col 2002 y 2004), existen sin embargo, pocos estudios que indiquen las muestras más adecuadas para el cultivo en casos de infección natural (Marín y col 1996a, , Grillo y col 1997, Marín y col 1999) y ningún estudio que contemple las distintas situaciones epidemiológicas. Es necesario establecer si existen diferencias en cuanto a los tejidos de elección en función del "estatus" epidemiológico, ya que los resultados de los test diagnósticos pueden variar en las distintas situaciones epidemiológicas (Jacobson 1998 y 2000).

Brucella melitensis es una especie de crecimiento lento y difícil, sobre todo en un aislamiento primario. Dos son los medios más utilizados, Farrell y Thayer Martin, aunque son muy escasos los trabajos de evaluación de ambos en el caso de infecciones naturales. Marín describe una menor 
sensibilidad del Farrell frente a Thayer Martin, aunque es con el uso combinado de ambos cuando esta autora alcanzó las mayores tasas de detección. Sin embargo, en el trabajo citado no se describe la situación epidemiológica de los animales, hecho que puede ser clave para determinar la efectividad de cada medio. Las posibilidades de aislamiento en cada tejido están determinadas por la patocronia de la enfermedad, por ello hemos reunido los tejidos en grupos en función de su significación en la patogenia. Los linfonódulos (Lnn.) de la cabeza (mandibular, parotídeo y retrofaríngeo) serían, por su proximidad con la vía de entrada, los primeros en ser colonizados pero también los primeros de donde son eliminados los gérmenes con mayor rapidez (Alton 1985, 1990, European Union 2001, Elzer 2002). Si las brucelas superan la reacción defensiva en el linfonódulo craneal se diseminan por el torrente sanguíneo a otras localizaciones a los 10-22 días postinfección (Biggi 1956 citado por Durán 1998) o durante varios meses (Barberán y Blasco 2002). Para representar este subgrupo (tejidos indicativos de diseminación) se tomó muestra de bazo, Lnn. subilíaco, Lnn. hepático e hígado. Los tejidos indicativos de excreción representan aquellos implicados en la diseminación del germen al ambiente del rebaño. Al ser las vías de excreción de brucela la mamaria y la vaginal tras el parto o aborto, se tomaron muestras de mama, Lnn. mamarios, e hisopo vaginal (Alton, 1990, Grillo y col 1997, European Union 2001, OIE 2008). Se trató estadísticamente por separado el grupo "Linfonódulo mamario y mama", debido a que también está implicado en la fase de diseminación y puede ser también indicativo de cronificación del proceso e incluso del estado de portador inaparente (Alton 1985, European Union 2001, OIE 2008).

\section{MATERIAL Y MÉTODOS}

Se sacrificaron en matadero 92 ovejas adultas (> 18 meses) procedentes de 15 rebaños, sospechosas de infección brucelar (positivos a FC, ya que actualmente en Europa la prueba de FC es la oficial y aunque más pruebas serológicas harían aumentar la sensibilidad, nuestro objetivo principal era detectar animales "realmente infectados", para lo que la necropsia y cultivo son la prueba "gold standard"). Los animales provenían del programa de saneamiento español pero los análisis serológicos y microbiológicos se realizaron para este estudio por el equipo de investigación en el Centro Nacional de Referencia para las Brucelosis Animales.

Tras la encuesta epidemiológica se clasificaron como procedentes de Rebaños con Brucelosis Activa (A), con más del $5 \%$ de prevalencia FC durante tres años y/o abortos debidos a $B$. melitensis en el mismo período (56 animales de 5 rebaños); Rebaños con Brucelosis Crónica (C), 1 - 5\% de prevalencia FC durante tres años y ausencia de abortos por B. melitensis (31 animales de 5 rebaños); Rebaños con muy Baja prevalencia (LP), 0,1 - 1\% FC durante tres años y sin abortos brucelares ( 5 animales de 5 rebaños). Según el "estatus" vacunal detectamos 56 animales vacunados de 8 rebaños y 36 no vacunados de 7 rebaños. La vacuna empleada fue Rev 1 de aplicación conjuntival a los 3-6 meses de edad y 1-2 x 109 CFU/dosis. Al realizarse en animales jóvenes inmaduros en esta dosis y vía, la reacción serológica desaparece a los pocos meses, por lo que la aparición de un animal FC positivo implica sospecha de contacto con el germen de campo.

Se tomaron muestras de Linfonódulos mandibulares (Lnn.MD); Linfonódulos parotídeos (Lnn.PT); Linfonódulos retrofaríngeos medios (Lnn.RF); Linfonódulos mamarios (Lnn.M); Linfonódulos subilíacos (Lnn.SI) (también: prefemoral, probablemente mencionado en la literatura que hace referencia a brucelosis como precrural y crural); Linfonódulos hepáticos (Lnn.H); bazo (Bz); hígado (Hg); mama (Mm); hisopo vaginal (Es). La siembra, crecimiento de cepas e identificación de las mismas se efectuó según el protocolo de Alton (Alton y col 1988).

\section{ANÁLISIS ESTADÍSTICO}

Para la evaluación estadística de las diferencias observadas entre grupos se aplicaron la prueba de Ji-cuadrado para analizar proporciones; el Análisis de la varianza (ANOVA) y la prueba $t$ (Student) o en su caso, menos de 5 observaciones, la prueba $\mathrm{F}$ (Fisher) para analizar series de datos distribuidos normalmente. La cuantificación del grado de infección de la canal se realizó determinando el número de colonias por placa en cada aislamiento según el cuadro 2 y dividiendo la suma de todas las categorías por el número máximo posible (número de especímenes multiplicado por 5) y expresado en porcentaje (Verger y col 1995). Para la comparación del grado de infección entre los grupos se empleó el test no paramétrico de U según Mann y Whitney. El procesamiento de datos se ha realizado con la Hoja de Cálculo Excel, versión 5.0, y los análisis estadísticos con los paquetes Episcope (Frankena y col 1990) y Epiinfo, versión 2002 (Dean y col 1994).

\section{RESULTADOS}

Se aisló Brucella en 36 de los 92 animales muestreados (frecuencia relativa $(\mathrm{fr})=33,04 \%$ ). Todas las cepas fueron identificadas como Brucella melitensis biotipo 3. Ninguno de los aislamientos del estudio fue de la cepa vacunal, sino de cepas de campo. Se aisló en 29 animales pertenecientes a rebaños con Brucelosis Activa (A), (fr $=51,8 \%$ de los animales de este grupo), frente a $7(\mathrm{fr}=22,8 \%)$ incluidos en los rebaños con Brucelosis Crónica (C). La diferencia entre ambas cifras fue significativa $(\mathrm{P}=0,0080)$. También fue significativamente más frecuente el aislamiento en rebaños No Vacunados $(\mathrm{fa}=21, \mathrm{fr}=53,8 \%$ ) respecto al de Vacunados (frecuencia absoluta $(\mathrm{fa})=15$, fr $=26,8 \%$ ); ( $\mathrm{P}<0,0001)$. En los rebaños de muy baja prevalencia (LP): ( 5 animales pertenecientes a 5 rebaños) todos los animales resultaron negativos en el cultivo microbiológico. 
En el cuadro 1 se especifica el porcentaje de aislamientos en los distintos tejidos y grupos de tejidos según el tipo de animal. El tejido donde se detectó brucela en un mayor número de animales fue en linfonódulo mamario (29 animales, $\mathrm{fr}=78,4 \%$ de los positivos), seguido de mama ( 26 y fr $=70,3 \%)$ y bazo $(14 \mathrm{y} \mathrm{fr}=37,8 \%)$. El hisopo vaginal fue positivo en tan sólo 9 animales ( $\mathrm{fr}=24,3 \%$ ). Sólo un animal fue positivo a partir de muestras de hígado y 4 de linfonódulos hepáticos. El grupo de muestras formado por "Linfonódulo mamario y mama" fue positivo en 34 animales ( $\mathrm{fr}=91,9 \%$ de los positivos), porcentaje significativamente más elevado que el de los grupos citados como "tejidos indicativos de diseminación": hisopo y linfonódulos de la cabeza, los cuales no presentaron diferencias entre sí. Los "tejidos indicativos de diseminación" están muy estrechamente ligados a "Linfonódulo mamario y mama", ya que siempre que se aisló en el primero lo fue también en el segundo.

En ningún tejido se aisló el microorganismo en proporción significativamente mayor en un tipo de rebaño respecto al otro, excepto en el grupo Linfonódulos mamarios que era más frecuente en el tipo $\mathrm{A}$ (Brucelosis Activa) que en el C (Brucelosis Crónica) $(\mathrm{P}=0,0647)$ a nivel de significación $90 \%$. En cuanto a los grupos de tejidos no observamos diferencias significativas entre los diferentes tipos de rebaños, aunque los linfonódulos de la cabeza de los rebaños Vacunados y No Vacunados presentaron diferencias significativas a nivel de $90 \%(P=0,0680)$ entre los rebaños A y C. Por tanto, en todas las situaciones epidemiológicas descritas predominó el aislamiento a partir de mama y linfonódulo mamario de forma mucho más frecuente que en el resto de los grupos -cabeza, tejidos indicativos de diseminación e hisopo- $y$, entre estos tres, ninguno predominó sobre los demás.

\section{DISCUSIÓN}

Es comúnmente aceptada (Jacobson 1988, Jacobson 2000, OIE 2008) la necesidad de realizar estudios en animales infectados naturalmente y representativos de las distintas situaciones epidemiológicas. Consideramos que los animales sujetos a una infección experimental son homogéneos en edad y "estatus" inmunitario, infectados en etapas de alta susceptibilidad, con dosis elevadas y administradas directamente en la vía de entrada (mucosa conjuntival) y con cepas hipervirulentas. En la infección natural, por el contrario, todos estos parámetros son variables y desconocidos. Por tanto, las infecciones experimentales presentan

Cuadro 1. Resultados microbiológicos por tejidos y tipo de rebaño.

Microbiological results per tissue and flock type.

\begin{tabular}{|c|c|c|c|c|c|c|}
\hline Rebaños & $\bar{A}$ & $\mathrm{C}$ & LP & $\mathrm{NV}$ & $\mathrm{V}$ & Total \\
\hline $\mathrm{n}^{\mathrm{o}}(+)$ & $56(29)$ & $31(7)$ & $5(0)$ & $36(21)$ & $56(15)$ & $92(36)$ \\
\hline$\%$ & 51,8 & 22,6 & 0 & 58,3 & 26,8 & 39,1 \\
\hline \multicolumn{7}{|c|}{ Muestras de la cabeza } \\
\hline $\operatorname{lnn}$. RF. + (\%*) & $5(17,2)$ & $2(28,6)$ & 0 & $4(19,0)$ & $3(20,0)$ & $7(19,4)$ \\
\hline $\operatorname{lnn} . \mathrm{PT}+(\% *)$ & $9(31)$ & $1(14,3)$ & 0 & $8(38,1)$ & $2(13,3)$ & $10(27,8)$ \\
\hline $\operatorname{lnn} . \mathrm{MD}+(\% *)$ & $5(17,2)$ & $2(28,6)$ & 0 & $5(23,8)$ & $2(13,3)$ & $7(19,4)$ \\
\hline Total $+(\% *)$ & $11(37,9)$ & $2(28,6)$ & 0 & $10(47,6)$ & $3(20,0)$ & $13(36,1)$ \\
\hline \multicolumn{7}{|c|}{ Tejidos indicativos de diseminación orgánica } \\
\hline $\operatorname{lnn}$. SI. + (\%*) & $4(13,8)$ & $1(14,3)$ & 0 & $4(19,0)$ & $1(6,7)$ & $5(13,9)$ \\
\hline $\operatorname{lnn} . \mathrm{H}+(\% *)$ & $3(10,3)$ & $1(14,3)$ & 0 & $2(9,5)$ & $2(13,3)$ & $4(11,1)$ \\
\hline $\mathrm{Hg}+(\% *)$ & 0 & $1(14,3)$ & 0 & 0 & $1(6,7)$ & $1(2,8)$ \\
\hline $\mathrm{Bz}+(\% *)$ & $11(37,9)$ & $3(42,9)$ & 0 & $9(42,9)$ & $5(33,3)$ & $14(37,8)$ \\
\hline Total $+(\% *)$ & $11(37,9)$ & $3(42,9)$ & 0 & $9(42,9)$ & $5(33,3)$ & $14(38,9)$ \\
\hline \multicolumn{7}{|c|}{ Tejidos indicativos de excreción } \\
\hline $\operatorname{lnn} . \mathrm{M}+(\% *)$ & $22(75,9)$ & $7(100)$ & 0 & $18(85,7)$ & $11(73,3)$ & $29(80,6)$ \\
\hline $\mathrm{Mm}+(\% *)$ & $20(69,0)$ & $5(71,4)$ & 0 & $16(76,2)$ & $9(60,0)$ & $25(69,4)$ \\
\hline Es $+(\% *)$ & $8(27,6)$ & $1(14,3)$ & 0 & $6(28,6)$ & $3(20,0)$ & $9(25,0)$ \\
\hline Total $+(\% *)$ & $27(93,1)$ & $7(100)$ & 0 & $20(95,2)$ & $14(93,3)$ & $34(94,4)$ \\
\hline
\end{tabular}

* Los porcentajes se calculan como el número de animales positivos en cada tejido o grupo / número de positivos (B) X 100.

Inn. RF Linfonódulos retrofaríngeos medios; Inn. PT: Linfonódulos parotídeos; Inn. MD: Linfonódulos mandibulares; Inn. SI: Linfonódulos subilíacos. (también prefemoral); Inn. H: Linfonódulos hepáticos; Hg: Hígado; Bz: Bazo; Inn. M: Linfonódulos mamarios; Mm: mama; Es: hisopo vaginal. 
Cuadro 2. Determinación del grado de infección. Determination of the level of infection.

\begin{tabular}{cc}
\hline Grado de infección & $\mathrm{N}^{\mathbf{o}}$ de colonias por placa \\
\hline 0 & 0 \\
1 & $1-5$ \\
2 & $6-25$ \\
3 & $26-125$ \\
4 & $126-625$ \\
5 & $>625$ \\
\hline
\end{tabular}

cifras más elevadas que las obtenidas en nuestro estudio, debido probablemente a las condiciones controladas y a que, en condiciones de campo, las exposiciones al antígeno pueden ser continuas a muy baja dosis, sin capacidad de colonizar pero propiciando la producción de anticuerpos detectables.

Respecto a la infección natural, las cifras obtenidas en nuestro trabajo son menores que las descritas en la literatura (Marín y col 1996ª,b, Grillo y col 1997). Sin embargo, en estos estudios no se especifica la situación epidemiológica del rebaño de procedencia, que bien podrían ser rebaños con Brucelosis Activa dada la similitud de resultados con las ganaderías Tipo A de este estudio. Nuestros datos revelan la necesidad de describir la situación epidemiológica de los animales de referencia empleados en los estudios científicos (Jacobson 1998, Jacobson 2000) y la necesidad concreta de especificar el tipo de infección del rebaño en los estudios microbiológicos, ya que observamos diferencias significativas en el porcentaje de aislamientos entre rebaños A, C y LP. Por tanto, son detectados y sacrificados en el transcurso de las campañas de control y erradicación un mayor número de animales cultivo negativo en el caso de los rebaños con Brucelosis Crónica (C) que aquellos que presentan Brucelosis Activa (A).

En las brucelosis agudas se infecta un gran número de animales con elevadas dosis de microorganismos y coincidiendo con períodos de alta susceptibilidad, por lo que la posibilidad de aislamientos es muy elevada. En casos de brucelosis crónicas el porcentaje de animales peligrosos epidemiológicamente es menor (excretores activos) y se ha establecido un equilibrio entre las defensas y el germen, por lo que muchos animales, a pesar de entrar en contacto con las brucelas, son capaces de luchar efectivamente contra ellas, bien eliminándolas completamente o bien reduciendo el número de patógenos viables en el animal. En estas circunstancias es evidente que la posibilidad de aislamiento será muy baja. Sin embargo, en estos animales la reacción serológica es clara, por lo que son detectados como positivos en las campañas de saneamiento.

También es más frecuente el aislamiento en rebaños no vacunados que en aquellos vacunados. El efecto protector de la vacuna sobre el porcentaje de animales cultivo positivo está ampliamente demostrado en estudios efectuados sobre la infección experimental en ovinos (Fensterbank y col 1982, Verger y col 1995) y en caprinos (Fensterbank y col 1987, Villegas y col 1999), pero no así en casos de infección natural. En nuestro caso las cifras obtenidas (53,8\% para los rebaños no vacunados y $21,9 \%$ para los rebaños vacunados) nos corroboran esta afirmación, aunque los porcentajes sean menores por las especiales características de la infección experimental ya comentadas. La vacuna conjuntival en edades tempranas protege de la infección y desaparecen los falsos positivos en pocos meses, sin embargo la brucelosis sigue sin ser erradicada en España. Es evidente que debe asumirse que la protección no es completa a lo largo de toda la vida del animal, así como que los test de detección sérica necesitan revisión. También se sugiere que la reacción positiva puede ser debida a falta de especificidad de la prueba oficial y por tanto la confirmación microbiológica de los casos es fundamental.

La distribución orgánica de Brucella fue muy similar en todos los tipos, con predominio de mama y linfonódulo mamario seguido de bazo y linfonódulos de la cabeza. Se puede establecer por tanto que el patrón de diseminación

Cuadro 3. Índice de infección por tejidos y tipo de rebaño.

Infection index per tissue and flock type.

\begin{tabular}{|c|c|c|c|c|c|c|c|c|c|c|c|}
\hline \multicolumn{2}{|c|}{ Rebaños } & \multicolumn{3}{|c|}{$\begin{array}{l}\text { Muestras } \\
\text { de la cabeza }\end{array}$} & \multicolumn{4}{|c|}{$\begin{array}{l}\text { Tejidos indicativos de } \\
\text { diseminación orgánica }\end{array}$} & \multicolumn{3}{|c|}{$\begin{array}{l}\text { Tejidos indicativos } \\
\text { de excreción }\end{array}$} \\
\hline & Total & $\operatorname{lnn} \cdot \mathrm{RF}$ & $\operatorname{lnn} . \mathrm{PT}$ & $\operatorname{lnn} . \mathrm{MD}$ & $\operatorname{lnn}$.SI & $\operatorname{lnn} . H$ & $\mathrm{Hg}$ & $\mathrm{Bz}$ & $\operatorname{lnn} . \mathrm{M}$ & $\mathrm{Mm}$ & Es \\
\hline $\bar{A}$ & 12,5 & 10 & 13,3 & 6,4 & 7,6 & 3,4 & 0 & 13,8 & 40 & 35,2 & 9 \\
\hline $\mathrm{C}$ & 14,3 & 8,6 & 5,7 & 8,6 & 5,7 & 5,7 & 5,7 & 14,3 & 45,7 & 40 & 2,9 \\
\hline LP & 0 & 0 & 0 & 0 & 0 & 0 & 0 & 0 & 0 & 0 & 0 \\
\hline $\mathrm{T}$ & 13,57 & 9,4 & 11,4 & 6,7 & 6,5 & 3,8 & 1,1 & 13,5 & 40 & 35,7 & 8,1 \\
\hline
\end{tabular}

Inn. RF: Linfonódulos retrofaríngeos medios; Inn. PT: Linfonódulos parotídeos; Inn. MD: Linfonódulos mandibulares; lnn. SI: Linfonódulos subilíacos (también prefemoral); Inn. H: Linfonódulos hepáticos; Hg: Hígado; Bz: Bazo; lnn. M: Linfonódulos mamarios; Mm: mama; Es: hisopo vaginal; T Total + Animales positivos. 
orgánica es similar para todas las situaciones epidemiológicas descritas, aunque es evidente que las muestras donde más fácilmente se recupera el microorganismo son los productos del aborto y el flujo vaginal (Corbel 1985, Crespo 1985, Alton y col 1988, Alton, 1990, Crespo 1994, Marín y col 1996 ${ }^{\mathrm{b}}$, Villegas 1999, Barberán y Blasco 2002, OIE 2008). Sin embargo, para la confirmación del diagnóstico inmunológico en los planes oficiales de control y erradicación, el escaso número de estudios al respecto hace difícil determinar en qué órganos del animal adulto será más fácil aislar el germen. Marín y col (1996a) señalan la relación entre fase patocrónica y órgano seleccionado para el aislamiento. Así en fases crónicas se acantona en células del sistema mononuclear fagocitario (SMF) como los linfonódulos o bazo, donde será más probable su aislamiento. Por ello en la necropsia la toma de muestras habitual comprende los órganos del SMF (bazo y linfonódulos), útero de la hembra gestante o abortada y mama de la parida (Corbel 1985, Marín y col 1996 ${ }^{\text {b }}$, OIE 2008). Pero tal cantidad de muestras hacen inviable el empleo rutinario de esta técnica. Debemos considerar una muestra de elección fiable para confirmar la enfermedad a nivel de rebaño. Además existen discrepancias en cuanto a la frecuencia relativa de aislamientos en cada localización, debido tal vez a la falta de estudios en animales infectados naturalmente.

En animales infectados en forma experimental (Fensterbank y col 1985, Alton 1990, Crespo 1894) es probable que la tasa de aislamiento esté muy relacionada con el punto de inoculación, presentándose mayores rendimientos en los aislamientos efectuados a partir de los linfonódulos de la cabeza, isolaterales con la vía de entrada (Crespo 1994). Varios autores (Crespo, 1994, Villegas y col 1999) también describen el efecto de la distancia al punto de inoculación en ganado caprino. Por el contrario, los resultados de nuestro estudio aportan cifras menores para los linfonódulos craneales, señalando claramente la diferencia que existe entre la infección experimental y la natural.

Los trabajos sobre infección natural son muy escasos, destacando Marín y col $\left(1996^{\mathrm{b}}\right)$, quienes señalan el mayor porcentaje de aislamientos en la mama y el menor, paradójicamente, en útero (sólo un 20\%), siendo los linfonódulos retromamarios los más sensibles a la infección natural, destacando también los ilíacos y prefemorales. Sin embargo, no especifica el tipo de infección del rebaño, como ya ha sido discutido.

Los datos de nuestro estudio señalan que el conjunto tisular (linfonódulos de la cabeza) no puede ser considerado tejido de elección en la toma de muestras en los estudios sobre infección natural, debido a la reducida frecuencia de aislamientos (baja en los rebaños con brucelosis crónica y tan sólo media en las brucelosis activas) y a la dificultad de la toma de muestras en condiciones de campo o matadero en forma rutinaria.

El grupo "Linfonódulo mamario y mama" debe ser contemplado en su doble significación. Por un lado, como señalan varios autores (Alton 1985, Grilló y col 1997), la presencia de Brucella en esta localización indica riesgo epidemiológico de excreción. Por otro lado, debe considerarse esta localización como reservorio crónico de la infección (Alton 1985, European Union 2001). Los datos obtenidos en nuestro estudio corroboran ambos extremos (potencialidad excretora y cronificación hacia el estado de portador inaparente). Además permiten señalar a este conjunto "Linfonódulo mamario y mama" como los tejidos de elección en la toma de muestras complementaria a las campañas de saneamiento, tanto por el elevado porcentaje de aislamientos como por el hecho de ser la mayor fuente de "falsos negativos" serológicos. En efecto, los animales no detectados serológicamente en nuestro estudio (datos no mostrados) son en su mayoría positivos a mama + Linfonódulo mamario.

Destaca el 100\% de aislamientos positivos a partir de linfonódulos mamarios de animales pertenecientes a los rebaños con Brucelosis Crónica (C), lo que refuerza la hipótesis de que este tejido es el responsable del mantenimiento de la brucelosis en el rebaño.

En el índice de infección de la canal no observamos diferencias significativas entre los tipos de rebaño A y C. Un animal mantiene la infección con menos frecuencia en el Tipo C, pero cuando lo hace será con la misma intensidad que en el Tipo A.

Los tejidos más afectados (índice de infección más elevado) en todas las situaciones son la mama y el linfonódulo mamario ( $36,7 \%$ y $41,1 \%$, respectivamente; datos no mostrados) con diferencias significativas respecto a todos los demás. Son por tanto tejidos de multiplicación muy activa y una importante fuente de diseminación, tanto por el número de animales afectados como por la intensidad de la excreción.

Sin embargo, sí se aprecia una diferencia significativa entre el índice de infección en "Linfonódulo mamario y mama" entre animales procedentes de rebaños no vacunados y vacunados (46,67 y 26,62 con $\mathrm{P}=0,0147)$. $\mathrm{La}$ vacunación ejerce un efecto protector sobre el grado de multiplicación del germen, aunque sólo en estos tejidos, ya que en el resto (cabeza, tejidos indicativos de diseminación y el hisopo) las diferencias entre vacunados y no vacunados no son significativas.

La importancia del exudado vaginal como elemento diseminador es tal, que se ha descrito como la principal causa del mantenimiento de la infección en el rebaño (Corbel 1985, Crespo 1994, Marín y col 1996 , GarínBastuji y col 1998, Barberán y Blasco 2002, OIE 2008). En nuestro estudio los porcentajes de aislamiento a partir del hisopo son menores que en la mama, lo que enfatiza los hallazgos descritos en la literatura sobre éxitos menores de aislamiento en el útero que en la mama. La muestra obtenida mediante hisopo vaginal no parece pues adecuada para la confirmación del diagnóstico serológico.

En los tejidos indicativos de diseminación orgánica debe destacarse la baja tasa de animales positivos (14 animales, 
$37,8 \%)$ y el reducido índice de infección en todos ellos $(6,2)$, así como el hecho de que siempre van acompañados de aislamiento positivo en mama y/o linfonódulos mamarios. Son por tanto tejidos no recomendables para su análisis rutinario.

En el bazo se aísla Brucella en el 37,8\% de los animales con infección y exhibe un índice de infección de 13,9. Es el órgano del grupo de tejidos indicativos de diseminación donde más frecuentemente se detecta Brucella. El aislamiento en bazo siempre va acompañado de aislamiento en mama y/o linfonódulos. Existe poca concordancia con la literatura consultada y que hace referencia a infección experimental donde los porcentajes son siempre más elevados (Fensterbank y col 1982, Crespo 1994) en ganado ovino y según Villegas y col (1999) en cabras. Existe mayor coincidencia con los casos de infección natural descritos por Marín y col (1996a) los que señalaron un 25\% de bazos positivos en cabras y $36 \%$ en ovejas. Los datos obtenidos en nuestro estudio sugieren que el papel del bazo sería menor al establecido por los diferentes autores consultados. Por tanto, apuntamos que no es una muestra imprescindible en la realización de análisis microbiológicos complementarios a las campañas de saneamiento ganadero, a diferencia de lo señalado en la literatura mencionada.

Diversos autores (Alton y col 1988, Blasco y Gamazo 1994, Barberán y Blasco 2002) coinciden en la necesidad de utilizar medios selectivos para el cultivo de Brucella. En nuestro estudio utilizamos dos placas de medio Farell y otras dos de Thayer Martín por cada uno de los tejidos muestreados con el fin de aumentar la sensibilidad (Marín y col 1996ª,b, European Union 2001, OIE 2008). Debemos señalar que se han realizado pocos estudios comparativos de la eficacia de ambos medios para el crecimiento del género Brucella. Marín y col (1996ª) obtienen una mayor sensibilidad en la detección de brucelas, a partir de ovejas infectadas naturalmente, con el medio de Thayer Martin que con el medio de Farrell. En dicho estudio se detectaron 10 animales cuyas muestras fueron positivas en Thayer Martin y negativas en Farrell, mientras que el caso inverso se presentó en una sola ocasión. Nuestros resultados son muy distintos, aunque con la coincidencia básica de la mejor sensibilidad por el uso simultáneo de ambos medios. Obtuvimos 14 muestras donde el resultado fue positivo Farrell y negativo a Thayer Martin, en tanto que el resultado inverso sólo ocurrió a partir de un tejido. La concentración de sustancias inhibidoras presentes en el medio de Thayer Martin es menor para no disminuir el crecimiento brucelar, sin embargo, esto ha provocado en nuestro estudio un mayor número de placas contaminadas. Obtuvimos ambas placas de Thayer Martin altamente contaminadas a partir de 5 tejidos que resultaron cultivo brucelar positivo a Farrell, siendo sólo uno el caso contrario. Aunque la diferencia en sensibilidad y grado de contaminación es evidente entre ambos medios, en ningún tejido el grado de crecimiento (índice de infección) es distinto en los medios de Farrell o de Thayer Martin, ni tampoco el porcentaje de casos en que la eficacia de Farrell es superior a la de Thayer Martin, pero el caso contrario es significativamente diferente (Thayer Martin con mayor número de colonias), por lo que la concentración de elementos nutritivos parece adecuada, presentándose las discrepancias como probablemente debidas a la concentración de sustancias inhibidoras. Consideramos que debe profundizarse en el estudio de nuevos medios más sensibles y selectivos, aunque es evidente que el uso de Farrell y Thayer Martin de forma simultánea incrementa las posibilidades de aislamiento.

Como conclusiones podemos establecer que se aíslan brucelas con mayor frecuencia a partir de animales sospechosos de enfermedad brucelar (positivos a fijación del complemento) pertenecientes a rebaños con Brucelosis Activa que en los pertenecientes a rebaños con Brucelosis Crónica. En aquellos rebaños con muy baja prevalencia no fue posible el aislamiento. Estos datos corroboran la necesidad de estudiar técnicas diagnósticas más específicas, así como de complementar los datos serológicos con un estudio epidemiológico y microbiológico. También resulta imprescindible la descripción de la situación epidemiológica de los rebaños en los estudios realizados sobre casos de infección natural.

La toma de muestras para complementar o confirmar los resultados obtenidos por las técnicas serológicas en el transcurso de las Campañas de Control y Erradicación de la Brucelosis debe incluir necesariamente tejido y linfonódulo mamario. Otros tejidos como linfonódulos craneales, bazo, linfonódulos hepáticos y subilíacos, hígado o hisopo vaginal aportan poca información adicional, por lo que su análisis rutinario no se considera necesario para la confirmación de la infección a nivel de rebaño.

La vacunación ejerce un efecto protector sobre la intensidad de la infección, mostrada como índice de infección de la canal y de los tejidos, pero no sobre la distribución orgánica del germen.

El uso simultáneo de los medios de Farrell y Thayer Martin es imprescindible para alcanzar la máxima eficacia en el aislamiento de Brucella.

\section{RESUMEN}

El sacrificio de animales seropositivos para el control de la brucelosis ovina requiere del aislamiento microbiológico como técnica de confirmación del proceso. Debido a la dificultad de realización de las técnicas microbiológicas en un elevado número de animales, así como de la gran cantidad de muestras, es necesario establecer qué tejidos son los más adecuados para ello. No existen datos al respecto obtenidos de animales infectados naturalmente y representativos de las distintas situaciones epidemiológicas tal y como recomienda la Organización Internacional de Epizootias. En nuestro trabajo realizamos la necropsia de 92 animales sospechosos de infección brucelar efectuando posteriormente el cultivo e identificación microbiológica de 10 tejidos diferentes. Los animales fueron clasificados en función de una encuesta epidemiológica como pertenecientes a Rebaños con Brucelosis Crónica, Activa o de 
muy Baja Prevalencia. Los resultados muestran diferencias en función del tipo de rebaño, así como indican que la toma de muestras de mama y linfonódulos mamarios es la más adecuada para la confirmación de la infección en un rebaño sospechoso. El uso combinado de los medios de cultivo Farrell y Thayer Martin mostró una mayor eficacia según los resultados obtenidos por nosotros.

\section{AGRADECIMIENTOS}

Agradecemos la colaboración tanto del Departamento de Agricultura de la Generalitat de Catalunya como de los veterinarios Delmir Prieto y Andreu Gil.

\section{REFERENCIAS}

Alton GG. 1985. The epidemiology of Brucella melitensis in sheep and goats. In: Verger JM, Plommet M (eds). Brucella melitensis, $a$ CEC seminar. Martinus Nijhoff, Dordrecht-Boston-Lancaster, The Netherlands, Pp 187-196.

Alton GG, LM Jones, RD Angus, JM Verger. 1988. In: INRA (eds). Techniques for the brucellosis laboratory. Institut National de la Recherche Agronomique. Paris, France.

Alton GG. 1990. Brucella melitensis. In: Nielsen K, Duncan JR (eds). Animal brucellosis. CRC Press, Boston, USA, Pp 383-409.

Barberán M, JM Blasco. 2002. Epidemiología, Patogenia y cuadro clínico, Brucelosis ovina. Ovis 82, 39-53.

Biggi P. 1956. Indagini sulla batteriemia brucellare in pecore sperimentalmente infettate. Ann Fac Med Vet Pisa 9, 264

Blasco J, C Gamazo. 1994. Brucelosis animal. Investigación y ciencia $218,56-62$

Corbel MJ. 1985. Bacteriological procedures in the diagnosis of Brucella infection. In: Verger JM, Plommet M (eds). Brucella melitensis a CEC seminar. Martinus Nijhoff, Dordrecht-Boston-Lancaster, The Netherlands, Pp 105-122.

Corbel MJ. 1997. Brucellosis: An overview. Emerg Infect Dis 2, 213-221.

Crespo F. 1985. Contribución al estudio de la etiología y la epidemiología de la brucelosis en España. Tesis Doctoral, Facultad de Veterinaria, Universidad Complutense Madrid, Madrid, España.

Crespo F. 1994. Brucelosis ovina y caprina. Office International des Epizooties, Paris, France.

Dean AG, JA Dean, D Coulombier, KA Brendel, DC Smith, AH Burton. 1994. Epi Info Version 6: A Word Processing, Database, and Statistics Program for Epidemiology on Microcomputers. Centers for Disease Control, Atlanta, USA.

Durán M. 1998. Comparación entre métodos inmunológicos de diagnóstico de la brucelosis ovina por Brucella melitensis y eficacia de la inmunización de ovejas adultas con la vacuna Rev-1 por vía conjuntival. Tesis Doctoral, Facultad de Veterinaria, Universidad de Murcia, Murcia, España.

Durán-Ferrer M, J Mendoza, A Osuna, V Caporale, A Lucas, L León, F Garrido. 2002. Evaluation of a new immunocapture test for the diagnosis of ovine brucellosis caused by Brucella melitensis. Vet Rec 151, 629-635.

Durán-Ferrer M, L Léon, K Nielsen, V Caporale, J Mendoza, A Osuna, A Perales, P Smith, C De-Frutos, B Gómez-Martín, A Lucas, R Chico, OD Delgado, JC Escabias, L Arrogante, R Díaz-Parra, F Garrido. 2004. Antibody response and antigen-specific gammainterferon profiles of vaccinated and unvaccinated pregnant sheep experimentally infected with Brucella melitensis. Vet Microbiol 100, 219-231.

Elzer PH, SD Hagius, DS Davis, VJ Delvecchio, FM Enright. 2002. Characterization of the caprine model for ruminant brucellosis. Vet Microbiol 90, 425-431.

European Union Commission. Scientific Committee on Animal Health and Animal Welfare. 2001. Brucellosis in Sheep and Goats (Brucella melitensis). Doc SANCO. C.2/AH/R23/2001. European Commission, Brussels, Belgium.

Fensterbank R, P Pardon, J Marly. 1982. Comparison between subcutaneous and conjunctival route of vaccination with Rev. 1 strain against Brucella melitensis infection in ewes. Ann Rech Vet 13, 295-301.

Fensterbank R, P Pardon, J Marly. 1985. Vaccination of ewes by a single conjunctival administration of Brucella melitensis Rev. 1 vaccine. Ann Rech Vet 16, 351-356.

Fensterbank R, JM Verger, M Grayon. 1987. Conjunctival vaccination of young goats with Brucella melitensis strain Rev 1. Ann Rech Vet 18, 397-403.

Frankena K, JP Noordhuizen, P Willeberg, PF Voorthuysen, JO Goelema. 1990. EPISCOPE: computer programs in veterinary epidemiology. Vet Rec 126, 573-57.

Garin-Bastuji B, JM Blasco, M Grayon, JM Verger. 1998. Brucella melitensis infection in sheep: present and future. Vet Res 29, 255-274.

Grillo MJ, CM Marin, M Barberán, JM Blasco. 1997. Transmission of Brucella melitensis from sheep to lambs. Vet Rec 140, 602-605.

Jacobson RH. 1988. Validation of serological assays for diagnosis of infectious diseases. Rev Sci Tech Off Int Epiz 17, 469-486.

Jacobson RH. 2000. Principles of validation of diagnostic assays for infectious diseases. In: Office International des Epizooties. Manиal of Standards for Diagnostic Tests and Vaccines. Paris, France, Pp 15-23.

Marín CM, JL Alabart, JM Blasco. 1996ª . Effect of antibiotics contained in two Brucella selective media on growth of Brucella abortus B. melitensis and Brucella ovis. J Clin Microbiol 34, 426-428.

Marín CM, MP Jiménez De Bagüés, M Barberán, JM Blasco. 1996 . Comparison of two selective media for the isolation of Brucella melitensis from naturally infected sheep and goats. Vet Rec 38, 409-411.

Marín, CM, E Moreno, I Moriyón, R Díaz, JM Blasco. 1999. Performance of competitive and indirect enzyme-linked immunosorbent assays, gel immunoprecipitation with native hapten polysaccharide, and standard serological tests in diagnosis of sheep brucellosis. Clin Diagn Lab Immunol 6, 269-272.

OIE, Office International des Epizooties. 2008. Caprine and ovine brucelosis (excluiding Brucella ovis). In: Manual of Diagnostic Tests and Vaccines for Terrestrial Animals 2008. $6^{\text {th }}$ ed. Office International of Epizooties, Paris, France, Pp 974-982.

Orduña A, A Almaraz, A Prado, MP Gutiérrez, A García-Pascual, A Dueñas, M Cuervo, R Abad, B Hernández, B Lorenzo, MA Bratos, A Rodríguez-Torres. 2001. Evaluation of an immunocaptureagglutination test (Brucellacapt) for the serodiagnosis of human brucellosis. J Clin Microbiol 38, 4000-4005.

Verger JM, M Grayon, E Zundel, P Lechopier, V Olivier-Bernardin. 1995. Comparison of the efficacy of Brucella suis strain 2 and Brucella melitensis Rev.1 live vaccines against a Brucella melitensis experimental infection in pregnant ewes. Vaccine 13, 191-196.

Villegas AH, AL Hernández, AE Díaz, GF Suárez. 1999. Bacteriología y fagotipificación de Brucella spp. aislada de caprinos y bovinos. Téc Pec México 37, 63-70. 